\title{
Chylothorax: An unusual manifestation of a large atrial septal defect
}

\author{
Carmelo Mignosa, MD, ${ }^{a}$ Vincenzo Duca, MD, ${ }^{\text {b }}$ Giuseppe Ferlazzo, MD, ${ }^{a}$ Innocenzo Bianca, MD, ${ }^{a}$

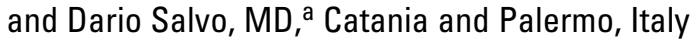

$\mathrm{A}$ ccumulation of chyle in the pleural space is a rare disorder that leads to metabolic disturbances and malnutrition. For its treatment, either conservative (medium-chain triglyceride [MCT] diet) or interven-

From the Divisione di Cardiochirurgia Azienda Ospedali Vittorio Emanuele, Ferrarotto, S. Bambino-Cattedra di Cardiochirurgia, Scuola di Specializzazione in Cardiochirurgia, Università di Catania, ${ }^{\mathrm{a}}$ Catania, Italy, and Unità Terapia Intensiva Neonatale Azienda Ospedale V. Cervello, ${ }^{\mathrm{b}}$ Palermo, Italy.

Received for publication April 2, 2001; accepted for publication April 13, 2001.

Address for reprints: Carmelo Mignosa, MD, Via Montello, 1, 95129, Catania, Italy (E-mail: mignosa@ctonline.it).

J Thorac Cardiovasc Surg 2001;122:1252-3

Copyright $\odot 2001$ by The American Association for Thoracic Surgery

$0022-5223 / 2001 \$ 35.00+0 \quad \mathbf{1 2 / 5 4 / 1 1 6 4 6 8}$

doi:10.1067/mtc.2001.116468 tional procedures (thoracic ductus ligation, pleurodesis) have been proposed. ${ }^{1,2}$

We describe the case of a 3-month-old baby with recurrent spontaneous chylothorax and a large atrial septal defect (ASD) in whom patch closure of the ASD alone successfully controlled the chylous effusion.

\section{Clinical Summary}

The patient was born at 36 weeks' gestation by cesarean delivery because a right pleural effusion was visualized by fetal echogram. Her birth weight was $2.8 \mathrm{~kg}$ with an Apgar score of 6 at 1 minute and 9 at 5 minutes. After birth the diagnosis of right pleural effusion was confirmed. She needed ventilatory support because of respiratory distress. Clear fluid, $100 \mathrm{~mL}$, was drained from the right pleural cavity. After 48 hours she was extubated and started on enteral feeding. Two days after institution of a normal diet, signs of respiratory distress recurred. A chest x-ray film showed a new pleural effusion, and milky fluid was drained from the pleural 
space. The presence of fat globules, chylomicrons, and triglycerides (concentration greater than $110 \mathrm{mg} / \mathrm{dL}$ ) in the fluid confirmed the diagnosis of chylous effusion. The baby was started on an MCT diet. Full clinical evaluation excluded any associated disease apart from a large ASD diagnosed by echocardiogram. On day 25 after birth she was discharged to her home in a good condition.

At 2 months of age a new right pleural effusion was detected and monitored regularly by chest $\mathrm{x}$-ray films. Because of progression despite the MCT diet and failure to thrive at 3 months of age, she was referred to us for surgery. On admission, she weighed $3.8 \mathrm{~kg}$ and was feeding poorly. The chest x-ray film revealed a large right pleural effusion. An echocardiogram showed situs solitus with normal atrioventricular and ventriculoarterial connections. A large secundum ASD was present. Through a midline sternotomy, cardiopulmonary bypass was instituted and the ASD was closed with an autologous pericardial patch. Before the chest was closed, the right pleural cavity was explored. The pleural space was drained, but it was not possible to find a clear site of leakage. The chest was then closed in the usual fashion, with a drain left in the right pleural space.

Mechanical ventilation was continued electively for 48 hours after the operation with pressure control ventilation, positive endexpiratory pressure, and higher than usual mean airway pressure, as proposed recently by Ragosta and Alfieris. ${ }^{3}$ The pleural tube did not produce drainage in the postoperative period. It was kept in place for 48 hours after feeding was restarted. She was started on a normal diet (full strength milk), building the input progressively to her normal requirement. On the eighth postoperative day she was discharged. At 6 months' follow-up she was thriving and no effusion was detected on chest radiography.

\section{Comment}

Leakage of lymph into the pleural cavities is a rare disorder that can cause severe malnutrition and deterioration of a patient's general condition. Chylothorax is rarely congenital or spontaneous, observed as a manifestation of lymphangiomatosis or thyrotoxicosis. ${ }^{4}$ Often it is due to direct injury or trauma to the thoracic duct. Most frequently it is a postoperative complication in cardiac surgery, mainly after operations for congenital heart defects. In this case, it is mainly due to thrombosis or high venous pressure in the superior vena cava. ${ }^{5}$
We report a case of chylothorax as a symptom of a large left-toright atrial shunt. Increased venous pressure in the right atrium created the hemodynamic basis for a hypertensive thoracic duct that led to chyle leakage and accumulation in the right pleural space. This was the rationale to treat the patient only by ASD closure after the initial dietary management was unsuccessful. The patient was referred to us for pleurodesis but, in this particular condition, we believed that would have been the wrong treatment. In fact, chylothorax was an unusual (never reported in literature previously) manifestation of the underlying intracardiac lesion.

Postoperative management is another crucial part of the treatment. As recently proposed by Ragosta and Alfieris, ${ }^{3}$ increasing mean airway pressure by pressure control ventilation and positive end-expiratory pressure has been demonstrated to be an effective therapeutic intervention in children with persistent chylous effusion. In combination with the surgical option adopted, this postoperative ventilatory strategy played a role in the management of the case.

\section{Conclusion}

Persistent chylothorax as a first manifestation of a large left-toright shunt at the atrial level resulting from a secundum ASD has never been described previously. The pleural effusion resolved after treatment of the intracardiac lesion without any associated procedure such as pleurodesis. Increased mean airway pressure by pressure control ventilation and positive end-expiratory pressure should be part of the postoperative treatment in such cases.

\section{References}

1. Bond SJ, Guzzetta PC, Snyder ML, Randolph JG. Management of pediatric postoperative chylothorax. Ann Thorac Surg. 1993;56:469-72.

2. Fernandez Alvarez JR, Kalache KD, Grauel EL. Management of spontaneous congenital chylothorax: oral medium chain triglycerides versus total parenteral nutrition. Am J Perinatol. 1999;16:415-20.

3. Ragosta KG, Alfieris G. Chylothorax: a novel therapy. Crit Care Med. 2000;28:1208-9.

4. Ibrahim H, Asamoah A, Krouskop RW, Lewis D, Webster P, Pramanik AK. Congenital chylothorax in neonatal thyrotoxicosis. J Perinatol. 1999;19:68-71.

5. DeBeer HG, Mol MJ, Jansen JP. Chylothorax. Neth J Med. 2000;56:25-31. 